\title{
BMJ Open Study protocol of a pilot study evaluating feasibility and acceptability of a psychosexual intervention for couples postallogeneic haematopoietic stem cell transplantation
}

\author{
Brindha Pillay (1) , ${ }^{1}$ Maria Ftanou, ${ }^{1,2}$ David Ritchie, ${ }^{3}$ Yvonne Panek-Hudson, ${ }^{3}$ \\ Michael Jefford, ${ }^{4,5}$ Teresa Garcia, ${ }^{3}$ Cassandra Shields, ${ }^{6,7}$ Jo Gniel, ${ }^{8}$ \\ Jo Phipps-Nelson, ${ }^{5,9}$ Allison Drosdowsky, ${ }^{9}$ Sarah Blaschke, ${ }^{9}$ Steve Ellen ${ }^{1}$
}

To cite: Pillay B, Ftanou M, Ritchie D, et al. Study protocol of a pilot study evaluating feasibility and acceptability of a psychosexual intervention for couples postallogeneic haematopoietic stem cell transplantation. BMJ Open 2020;10:e039300. doi:10.1136/ bmjopen-2020-039300

- Prepublication history for this paper is available online. To view these files, please visit the journal online (http://dx.doi. org/10.1136/bmjopen-2020039300).

Received 10 April 2020 Revised 14 September 2020 Accepted 11 October 2020
Check for updates

(C) Author(s) (or their employer(s)) 2020. Re-use permitted under CC BY-NC. No commercial re-use. See rights and permissions. Published by BMJ.

For numbered affiliations see end of article.

Correspondence to Dr Brindha Pillay; brindha.pillay@petermac.org

\section{ABSTRACT}

Introduction Sexual dysfunction is one of the most common side effects of allogeneic haematopoietic stem cell transplantation (HSCT) for haematological cancers. Problems can persist between 5 and 10 years post-transplant and impact mood, couple intimacy and relationship satisfaction. Few intervention studies, however, target sexual dysfunction in patients postHSCT. This pilot study aims to examine the feasibility and acceptability of implementing a psychosexual intervention for HSCT survivors and their partners posttransplantation.

Methods and analysis Fifteen allogeneic HSCT survivors and their partners will be recruited. Patients who are more than 3 months post-transplantation will be sent invitation letters describing the couples' psychosexual intervention that will be offered through this study. The intervention will comprise two components: (1) psychosexual education about medical and behavioural treatment options for sexual dysfunction delivered by a haematology nurse consultant; (2) emotionally focused therapy-based relationship education programme for couples delivered by a clinical psychologist (four sessions of 1.5 hours each). Couples who consent to participate will be administered a series of measures assessing mood, relationship satisfaction and sexual dysfunction preintervention and post-intervention, as well as satisfaction with the intervention postintervention. Feasibility of the intervention will be examined via recording enrolment rate, adherence, compliance with completing outcome measures and fidelity of intervention delivery.

Ethics and dissemination Ethics approval has been obtained at the Peter MacCallum Cancer Centre in Melbourne, Australia. Results will be presented at national and international conferences and published in a peerreviewed journal so that in can be accessed by clinicians involved in the care of allogeneic HSCT patients. If this intervention is found to be feasible and acceptable, its impact will be examined in a future randomised controlled trial and subsequently implemented as part of routine care in the allogeneic HSCT population.
Strengths and limitations of this study

- This is one of the first studies examining the feasibility and acceptability of delivering a multidisciplinary (medical and psychosocial) psychosexual intervention to couples postallogeneic haematopoietic stem cell transplantation (HSCT).

- The four-session relationship education programme component of the intervention has a theoretical basis in emotionally focused therapy for couples, which is known to have a good evidence base in non-cancer groups but has not yet been trialled in a HSCT population.

- Patients and their partners will be recruited from a single institution, thus potentially limiting uptake rates and applicability of findings to the broader HSCT population.

- Relatively small numbers.

\section{INTRODUCTION}

Allogeneic haematopoietic stem cell transplantation (HSCT) is a treatment that is used for some patients with haematological malignancies. As part of the treatment, patients undergo high doses of chemotherapy, followed by infusion of blood stem cells from a donor. HSCT survivors are at risk of developing graft-versus-host disease (GVHD) where the body is attacked by donor immune cells. ${ }^{1}$ Chronic GVHD is managed by administering immunosuppressants that suppress the immune system's response but can also result in various complications. ${ }^{2}$ Patients who receive HSCT treatment are often under the age of 50 years and relatively healthy prior to their diagnosis. Thus, many women receiving treatment are premenopausal, and many women and men may have active sexual lives prior to commencing treatment. ${ }^{2}$ 
Sexual dysfunction is one of the most commonly experienced issues following allogeneic HSCT $^{2}$ due to the effects of chemotherapy, total body irradiation and medications for chronic GVHD. Treatment agents are toxic to gonadal functions and can lead to genital tissue sensitivity, atrophy or scarring. ${ }^{2}$ In a recent Australian study of 421 allogeneic HSCT patients, a large percentage of men reported erectile dysfunction (79\%) and decreased libido (61\%), while women reported loss of libido (83\%), painful intercourse $(73 \%)$ and less enjoyment of sex $(68 \%)$, as well as vaginal dryness $(73 \%){ }^{3}$ Genital tract GVHD is another known common complication for women. ${ }^{4}$ Some survivors continue to experience problems up to 5-10 years post-HSCT. ${ }^{5}$ As survival rates post-HSCT have increased, it is important to address the long-term impacts on survivors' sexual quality of life.

Medical interventions for sexual dysfunction in this population include hormone therapy options, PDE5 inhibitors, vaginal dilators and vacuum erection devices. In addition to medical interventions for sexual dysfunction, studies have shown that it is essential to address the cognitive, emotional and relational factors that impact on sexual functioning. ${ }^{12}$ There are few intervention studies using a multidisciplinary approach (ie, medical management of symptoms and psychosocial counselling targeted at improving attachment security in couples) to improve sexual dysfunction in patients post-HSCT. The small number of existing interventions for cancer populations post-treatment have demonstrated mixed results and poor overall effectiveness, particularly for women. ${ }^{6}$

A recent pilot study from Boston explored the impact of a multimodal intervention on sexual function in allogeneic HSCT patients. Patients were at least 3 months posttransplantation and had indicated through screening that sexual dysfunction was causing them distress. The intervention comprised monthly visits that assessed the causes of sexual dysfunction, educated and empowered them to address their sexual concerns and provided them with interventions targeted to their needs. ${ }^{7}$ A significant proportion of the 47 patients who participated reported improvements in satisfaction, interest in sex, orgasm, erectile function, vaginal lubrication and vaginal discomfort from preintervention to postintervention. The intervention included a psychoeducation component aimed at addressing important issues such as depression, anxiety, body image concerns, loss of intimacy and problems with interpersonal relationships and communication. However, it is important to note that the intervention was targeted to the HSCT patients only (rather than to couples) and did not use a structured theoretical framework for delivering the psychosocial component of the intervention. It is essential to address patient sexual dysfunction within a couples' framework, given the complexity of sexual interactions and the relationship between sexual dysfunction and couple-related factors. ${ }^{8}$

Emotionally focused therapy (EFT) is a structured therapy based on attachment, systemic and humanistic theory. ${ }^{9}$ Research has consistently demonstrated EFT to improve relationship satisfaction, maintain improvements across time (see metanalysis by Beasley and Ager ${ }^{10}$ ) and support couples to develop a more secure bond from which to address difficult topics such as sexual problems. ${ }^{11}$ EFT provides a framework to enable the development of a secure attachment within dyads by creating a safe space in which couples are able to understand their primary emotions and convey them to their partners. ${ }^{12}$ When a patient and partner perceive each other as a 'safe haven', this creates the optimal conditions for healthy intimate relationships and sexual functioning. ${ }^{12}$ A detailed theoretical basis for how EFT for couples can improve their sexual relationship, via improving the attachment bond of the couple, has been documented by Johansen and Zuccarini. ${ }^{13}$

There is evidence to support positive outcomes for interventions with couples that use an EFT framework. ${ }^{14}$ These include improving relationship satisfaction, couple communication and treating sexual difficulties in non-cancer groups, including culturally diverse populations and same-sex couples. ${ }^{15}$ Improvement in relationship satisfaction was associated with increases in attachment security in couples across time as they underwent the EFT intervention. ${ }^{16}$ In one study, it was found that sexual satisfaction of couples increased from preintervention to postintervention and across follow-up in a period of 2 years. Furthermore, the authors reported that decreases in attachment avoidance from preintervention to postintervention predicted improvements in sexual satisfaction over time. ${ }^{17}$ With regards to EFT-based research in cancer populations, one randomised trial demonstrated an improvement in marital functioning and perceived caregivers' empathic behaviour in patients with advanced cancer following delivery of a modified EFT intervention for couples. ${ }^{18}$ However, sexual difficulties were not assessed in this study.

Few intervention studies examine the impact of a multidisciplinary intervention to assist couples in managing sexual dysfunction post-HSCT. This will be the first study to pilot a couples' intervention that combines the medical management of sexual dysfunction with a structured but brief EFT-based relationship education programme in an allogeneic HSCT population.

The aim of this study is to examine the feasibility and acceptability of implementing a five-session psychosexual intervention for HSCT survivors and their partners who are more than 3 months post-transplantation. This time frame was chosen as patients are likely to be experiencing less significant physical side effects of transplantation by that time. Furthermore, an intervention offered to patients at an early stage may be more effectively received compared with providing it at a much later stage posttransplant, when relationship patterns may be more ingrained. Given that some patients may need a longer period to recognise sexual concerns post-HSCT, no upper time limit post-transplantation was placed on inclusion of patients into the study. 


\section{METHODS AND ANALYSIS}

This study is a prospective, single-arm descriptive study of a novel psychosexual intervention with patient and partner measures administered preintervention and postintervention.

\section{Patient and public involvement}

Patient feedback will inform the delivery of this pilot intervention. In particular, a survey will be administered to patients and partners following intervention delivery to elicit feedback regarding their experience of the intervention, including the time burden and satisfaction with the two components of the intervention. The information elicited from patients and their partners will be used to refine the intervention prior to testing it in a randomised controlled trial.

\section{Participants}

Fifteen couples will be recruited into the study. This target number is in line with numbers that have been recruited in a previous pilot couples intervention for patients with cancer, across a similar time frame of 10 months. ${ }^{18}$ Study recruitment will commence in late 2020.

\section{Participant inclusion criteria}

Each participating couple must meet the following criteria to participate in the study:

- Aged $\geq 18$ years old (both patient and partner).

- More than 3 months posthaematopoietic transplantation to treat a haematological malignancy.

- Patient has not relapsed since transplant.

- In a sexual relationship (same-sex couples included) of $\geq 1$ year (as reported by the patient) but couples do not need to be currently sexually active to participate in the study.

- Able to give informed consent (ie, no psychiatric/ cognitive condition that would impact informed consent, as based on clinical judgement).

- Patient states that they have sexual health problems and would like help with these issues.

\section{Participant exclusion criteria}

Couples meeting any of these criteria will be excluded from the study.

- Currently receiving couples counselling from a therapist not involved in the study.

- Partner is not willing to participate in the intervention.

- Patient has had a relapse of disease and/or has been readmitted to hospital.

- Patient is suffering from significant ongoing side effects that might impede their participation.

\section{Participant recruitment and consent}

Participants will be recruited from Clinical Haematology at Peter MacCallum Cancer Centre and Royal Melbourne Hospital Allogeneic Transplantation Service. Site of recruitment will be the Peter MacCallum Cancer Centre where patients attend their outpatient follow-up post-transplantation visits. Eligible patients (ie, patients who meet inclusion and exclusion criteria above) who are more than 3 months post-transplantation will be identified by the post-transplant nurse consultant on the research team and sent an invitation letter about the study with a brief description of the psychosexual intervention. Patients will be given an option to decline further contact or to ask for further information by returning a reply slip via mail using a reply-paid envelope provided. The invitation letter will explain that if the research team do not hear back from the patient in 2 weeks, the patient will be contacted via phone to ascertain their interest in the study. If patients and their partners are interested in participating, they will be provided with verbal and written information on the project, including the participant information and consent form (PICF).

Signed consent will be provided in clinic by patients and partners who agree to participate when the study is introduced. Alternatively, patients will be mailed a copy of the PICF and asked to return the signed consent form in a reply-paid envelope.

\section{Intervention}

The pilot intervention will comprise two components:

1. Psychosexual education about medical and behavioural treatment options for sexual dysfunction.

2. EFT-based relationship education programme for couples.

\section{Psychosexual education}

A 60 min individual psychosexual education session will be delivered to couple participants by a specialist nurse. This session will include information about medical and behavioural management of sexual dysfunction (including the use of hormone treatment, topical treatments, lubricants and vaginal dilators for women, and phosphodiesterase type 5 inhibitor (PDE5) inhibitors). The session will be delivered using the Permission, Limited information, Specific Suggestions and Intensive Therapy model.

\section{EFT-based relationship education programme for couples}

The couples' relationship education programme will comprise four $90 \mathrm{~min}$ sessions and be delivered to each couple by a clinical psychologist. It will use an EFT framework, aimed to assist couples in building and enhancing the attachment bond to improve the quality of their relationship and enhance sexual intimacy. ${ }^{16}$ The intervention material was adapted from three EFT resources developed for couples ${ }^{19-21}$ and modified to make it relevant to the HSCT population.

In session 1, the goal will be to establish an alliance with the couple, to introduce the programme and to allow each of them the opportunity to talk about the different ways that cancer and the HSCT has affected their relationship.

Session 2 will focus on identifying negative interactional cycles and attachment vulnerabilities within the dyad that trigger such interactional patterns. The session will help couples to access underlying emotions supporting the 
reactive moves in the cycle and then reframe the problem in terms of the negative cycle (rather than each other). The couples will also be taken through an explanation about different types of vulnerable emotions that can trigger negative interactions as well as ways to communicate more effectively.

Session 3 will assist the couple in sharing fears and needs with each other in a manner that helps their partners respond to them. This will facilitate the deeper expression of attachment emotions and needs, creating more open emotional engagement within the couple. In addition, psychoeducation regarding the impact of cancer and HSCT on the sexual relationship will be provided.

Session 4 will aim to elicit discussion about the couple's sexual relationship, including previously formed perceptions of self, other and the relationship and how this can be related to sexual intimacy. Other topics of discussion will include adopting a flexible view of sexual intimacy after disease and treatment stressors and practical ways to continue to nurture the relationship moving forward. Finally, an overview of sessions will be provided. This EFT intervention aims to equip couples with skills to establish a more secure bond and create new positive interactions within the dyad, in the context of living with a haematological cancer and undergoing an intense treatment.

\section{Evaluation measures and data collection}

The measures, data collection and time/place of data collection, according to the project aims, are described below. Regular quality assurance will be undertaken during data collection to ensure accuracy, precision and completeness of study data.

Specifically, demographic data will be collected, and evaluation measures will be administered preintervention and postintervention to determine participant acceptability in completing the range of measures. In addition, feasibility of delivering the intervention will be assessed via a range of methods including assessing referral rates into the study, patient uptake of the intervention, patient adherence to the intervention and therapist fidelity in administering the intervention. Preliminary trends in the efficacy of intervention will be observed via examining differences in scores preintervention and postintervention.

\section{Patient demographic and medical record data}

After consenting to the project, the patient's demographic and clinical characteristics will be collected from the medical record and from their treating team. Data collected will include:

- Age of patient and partner.

- Sex of patient and partner.

- Haematological cancer characteristics.

- Transplant details, including whether there was incidence of graft-versus-host disease.

- Details such as ethnicity, marital status, education level and length of relationship will be obtained through patient verbal self-report during the assessment part of the counselling sessions.

\section{Measures}

Patient Health Questionnaire-9 (PHQ-9)

The PHQ-9 is a widely used measure of depression known to have excellent reliability and validity. ${ }^{22}$ Scores range from 0 to 27. Higher scores represent higher levels of depression. There are established cut-off points of 5, 10, 15 and 20, which indicate mild, moderate, moderately severe and severe depression, respectively. ${ }^{22}$

\section{General Anxiety Disorder-7 (GAD-7)}

The GAD-7 is a seven-item questionnaire measuring anxiety, which has strong psychometric properties. ${ }^{23}$ Scores range from 0 to 21. Higher scores represent higher levels of anxiety. There are established cut-off points of 5, 10 and 15, which indicate mild moderate and severe anxiety, respectively. ${ }^{23}$

\section{The Experiences in Close Relationship Scale - Short Form (ECR- short form)}

The ECR-short form is a 12-item measure that was developed from the ECR scale (Brennan et al 1998) ${ }^{24}$ by Wei and colleagues. ${ }^{25}$ Each item is answered based on a 7-point Likert scale, with higher scores indicating poorer attachment in the relationship. There are two scales: attachment anxiety and attachment avoidance. Validity of the short form version was found to be equivalent to the original version of the ECR. ${ }^{25}$

\section{Perceived Relationship Quality Components Inventory (PRQC)}

The PRQC measure is an 18-item scale used to measure relationship quality among couples. It has six domains: satisfaction, commitment, intimacy, trust, passion and love. ${ }^{26}$ Items are answered based on a 7-point Likert scale, with a higher score indicating better relationship quality. Confirmatory factor analyses indicated that the individual subscales are reliable. ${ }^{26}$

\section{International Index of Erectile Functioning (IIEF)}

The $\mathrm{IIEF}^{27}$ is a widely used 15 -item instrument to measure the five domains of male sexual function including erectile function, orgasmic function, sexual desire, intercourse satisfaction and overall satisfaction. A review demonstrated that the IIEF has sound psychometric properties, including test reliability and validity as well as a high degree of sensitivity and specificity. ${ }^{28}$ Outcomes on this measure correlate well with other measures of sexual function treatment outcomes. Scores range from 0 to 75 . Higher scores indicate better sexual function.

\section{Female Sexual Function Index (FSFI)}

The FSFI is a 19-item questionnaire that assesses female sexual function across five domains of desire, arousal, lubrication, orgasm, satisfaction and pain. ${ }^{29}$ High testretest reliability and internal consistency as well as construct validity was demonstrated in the assessment of female sexual function in clinical and non-clinical 


\begin{tabular}{lll}
\hline Table 1 & Measures pre intervention and post intervention \\
\hline Participant & $\begin{array}{l}\text { Pre } \\
\text { intervention }\end{array}$ & $\begin{array}{l}\text { Post } \\
\text { intervention }\end{array}$ \\
\hline Patient & $\times$ & $\times$ \\
\hline PRQC & $\times$ & \\
ECR-short form & $\times$ & $\times$ \\
PHQ-9 & $\times$ & $\times$ \\
GAD-7 & $\times$ & $\times$ \\
\hline FSFI/IIEF (male or female) & & $\times$ \\
\hline Patient Satisfaction Survey & & \\
Partner & $\times$ & $\times$ \\
GAD-7 & $\times$ & $\times$ \\
PHQ-9 & $\times$ & \\
ECR-Short form & $\times$ & $\times$ \\
\hline PRQC & & $\times$ \\
\hline Partner Satisfaction Survey & & \\
\hline
\end{tabular}

ECR-short form, experiences in close relationship scale - short form; FSFI, female sexual function index; GAD-7, general anxiety disorder-7; IIEF, international index of erectile functioning; PHQ-9, patient health questionnaire-9; $P R Q C$, perceived relationship quality components inventory.

samples. Scores range from 2 to 36 . Higher scores indicate higher level of sexual function. ${ }^{29}$

\section{Patient and Partner Satisfaction Survey}

This survey was designed by the research team to determine the patient's and partner's experience of the intervention, including what aspects they found helpful/ unhelpful and any changes in their relationship functioning following the intervention. This survey consists of 11 questions (a mix of multichoice and open-ended items) and is expected to take approximately $10 \mathrm{~min}$ to complete. Patients and partners will complete this survey within 2 weeks of completing the intervention, or earlier if they withdraw prior to completion of the intervention.

\section{Satisfaction survey after each session}

Patients and partners will be given a satisfaction survey after each of the four sessions within the relationship education component of the intervention. It comprises four questions briefly asking participants what was beneficial from sessions and what could have been done differently.

Table 1 indicates measures used preintervention and postintervention.

Figure 1 below demonstrates the study process and measures used at each time point.

\section{Feasibility of intervention}

To measure feasibility, the fidelity of the intervention will be assessed through: (1) a review of each session by the clinician administering the intervention using session checklists and (2) recording all psychological therapy sessions and reviewing $10 \%$ of these to check compliance with protocols.
Session checklists

The nurse and psychologist administering the treatment will complete a session checklist after each session to ensure that all topics are covered in the intervention delivery. The session checklist used by the psychologist will be based on the Session Outline that was developed for the 'Hold Me Tight' and 'Healing Hearts Together' programmes. ${ }^{19} 20$

\section{Session recordings}

With participant's consent, sessions delivered by the psychologist will be recorded using a small portable audio recorder and $10 \%$ of these will be selected randomly for review. Each audio recording will be assigned a unique identifier. Recordings will be assessed by an objective rater who will score the fidelity to the intervention using the session checklist and the EFT-Therapist Fidelity Scale. Audio recordings will be securely stored and only accessed by the principal investigator and two study investigators. At the conclusion of the study, the audio recordings will be destroyed. Participants can decline to have their session recorded with no impact on their treatment and or participation in the study.

\section{Referral rates, uptake and patient adherence}

A case report form (CRF) will be used by the project team to assess referral rates into the study, uptake of the intervention and patient adherence to the intervention. In particular, data will be collected regarding number of patients who were referred to the study, those that refused intervention despite meeting eligibility criteria (including reasons for declining participation) and number of intervention sessions completed.

\section{Clinicians and project team time}

Time taken to deliver each component of the intervention as well as follow-up care (eg, follow-up phone calls and referrals following intervention) will be collected on the CRF by recording number of minutes or hours spent on each task.

\section{Data analysis}

Demographic, clinical and feasibility data (including time measures) and survey responses will be analysed descriptively (count/percentage, mean/SD, median/IQR as appropriate). Patient-reported and partner-reported outcomes will be scored according to their manuals and will be presented descriptively. Preliminary analyses comparing preintervention and postintervention outcomes will be conducted using paired t-tests. Feasibility criteria (see table 2) and data collected will be based on previous studies. ${ }^{30} 31$ The Patient/Partner Satisfaction Surveys will also be used to assess the impact of the intervention and its feasibility by reporting frequency data for each item.

Data will be managed through REDCap, and quantitative data will be analysed using SPSS (V.24). Free-text items from the patient satisfaction surveys will be analysed using summarising content analysis using NVivo software. A 


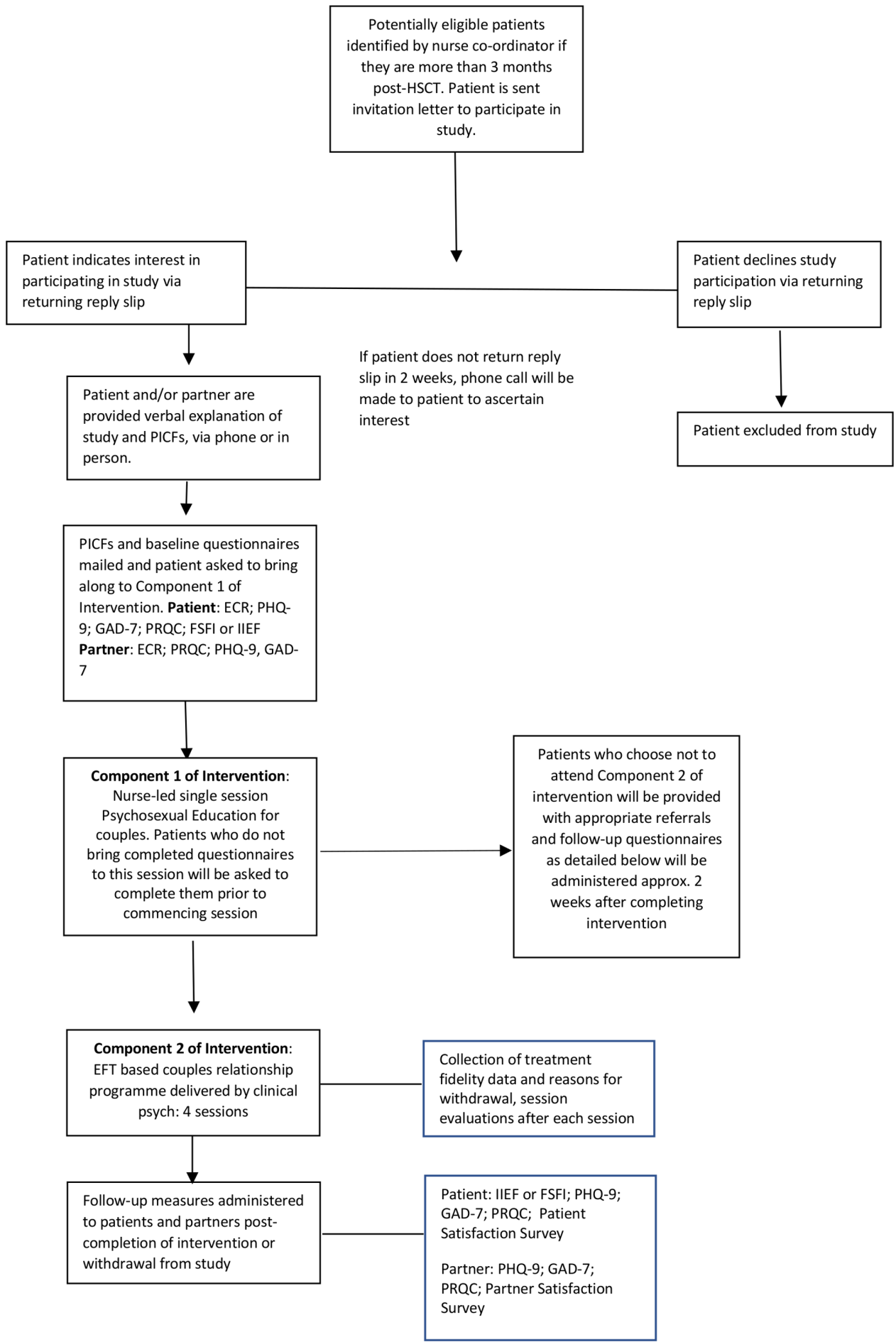

Figure 1 Study process and measures used at each time point. ECR, experiences in close relationship scale; EFT, emotionally focused therapy; FSFI, female sexual function index; GAD-7, general anxiety disorder-7; HSCT, haematopoietic stem cell transplantation; IIEF, international index of erectile functioning; PICFs, participant information and consent forms; PHQ-9, patient health questionnaire-9.

deductive content analysis approach will be used for coding data. Predefined categories will be formulated based on the research questions informing the study. Additional inductive codes will be identified from the survey responses.

\section{Ethics and disemmination}

This study, protocol and all instruments, including the informed consent document, have been approved by the
Human Research Ethics Committee (HREC) of the Peter MacCallum Cancer Centre (HREC reference number: HREC/53351/PMCC-2019; Project number: 19/54).

\section{Data storage and privacy issues}

A unique study identification number system will be used for any data collected. This system involves keeping a 'key' that specifies and links the patient's personal 
Table 2 Feasibility criteria for study

\begin{tabular}{|c|c|c|c|}
\hline Outcome & Value & $95 \% \mathrm{Cl}$ & Feasibility criteria \\
\hline Enrolment rate & 15 of $38(39 \%)$ & $24 \%$ to $57 \%$ & At least $39 \%$ of eligible patients (and their partners) will be enrolled. \\
\hline Adherence & 10 of $15(67 \%)$ & $38 \%$ to $88 \%$ & $\begin{array}{l}\text { At least } 67 \% \text { of patients (and their partners) enrolled in the study will } \\
\text { attend four intervention sessions. }\end{array}$ \\
\hline $\begin{array}{l}\text { Compliance with } \\
\text { assessments }\end{array}$ & 12 of $15(80 \%)$ & $52 \%$ to $96 \%$ & $\begin{array}{l}\text { At least } 12 \text { of } 15 \text { patients (and their partners) will complete the main } \\
\text { study measures (PRQC and FSFI/IIEF) postintervention. }\end{array}$ \\
\hline Fidelity & $\begin{array}{l}\text { This will depend on number } \\
\text { of sessions delivered for each } \\
\text { couple, thus a value has not been } \\
\text { entered at this stage. }\end{array}$ & & $\begin{array}{l}\text { At least } 90 \% \text { of the psychology interventions sessions delivered } \\
\text { will cover all required material based on the session checklist. An } \\
\text { individual rater would use the EFT-Therapist Fidelity Scale (a 3-point } \\
\text { Likert scale). }{ }^{2627}\end{array}$ \\
\hline
\end{tabular}

EFT, emotionally focused therapy; FSFI, female sexual function index; IIEF, international index of erectile functioning; PRQC, perceived relationship quality components inventory.

identifying information (eg, names and Unit Record Number (URNs)) with the patient's and partner's corresponding study identification number (eg, PT01/ PR01). The key will be kept electronically (in a passwordprotected Excel spread sheet) separate from all hardcopy and softcopy data. Electronic data will be stored in password-protected folders on Peter Mac's secure servers. Identifying information (ie, patient's name and contact details) will be obtained from the medical record and/ or consent form only for the purposes of contacting the patient. This information will not be used in data analysis and will be removed from the database at the conclusion of the project. Survey data will be entered into REDCap, a secure database.

Only members of the project team will have access to these data, in accordance with the National Statement on Ethical Conduct in Human Research 2007 and the Australian Code for Responsible Conduct of Research 2018. Hardcopy data will be stored in locked filing cabinets within Peter Mac Department of Psychosocial Oncology. Five years after publication or dissemination of project outcomes, hard copy and electronic data will be destroyed.

\section{Withdrawal criteria}

It is not expected that patients will be withdrawn by the research team or therapist involved in delivering the intervention, as the intervention schedule may be modified, depending on patient needs. If patients require referral to other practitioners for complementary care (eg, medication), or care for unrelated morbidity, this will be recorded on the database.

Patients who opt to withdraw from the study will be asked if they consent to completing the follow-up measures and for their existing data to be included in analyses. If consent is not given for the latter, their data will be erased from the database. Any electronic or paper records pertaining to their involvement will be destroyed at the completion of the study, with the exception of medical notes entered into the electronic system. A record of patients who have withdrawn from the study will be maintained in a secure database until the completion of the study to ensure that these patients are not approached again by the project team. Patients will be unable to withdraw their data after the completion of the study as their data may have already been used in analyses.

\section{Confidentiality}

It is not expected that participating in this project will pose any risks of harm to participants. If any disclosures of risks to safety (eg, suicidal ideation) occur during any stages of the project, standard clinical processes will be followed including safety planning with the participant and when needed advising an appropriate support person such as a member of the participant's treating team and/or a family member. This limit to confidentiality is included in the PICF.

\section{Safety reporting}

The potential for adverse events is deemed to be low in this study. In the event that a member of the study team observes any significant distress during their interaction with the couple, or items endorsed on psychological measures indicate high levels of distress (eg, endorsing thoughts of dying and self-harm on PHQ-9), he or she will offer an appropriate referral to a psychologist, psychiatrist or other relevant healthcare professional. The couples' intervention can continue or be aborted depending on the response of the participant. Questionnaire responses will be scored within three working days of being received to ensure that any high distress scores from questionnaire data will be reported to the treating clinician and/or nurse coordinator in a timely manner. Participants may then be referred to receive treatment from a qualified psychologist.

The sponsor and ethics department will be notified immediately of any safety issues and the management of these, respectively.

\section{Dissemination}

This study will be registered with the Australia New Zealand Clinical Trials Registry. Results obtained from the study will be published in peer-reviewed journals and inform the development of a randomised controlled trial and delivery of the intervention in routine clinical practice. Study findings will be disseminated to clinicians 
involved in the care of allogeneic HSCT patients through presentations to relevant departments within the institution and at national and/or international conferences.

\section{Author affiliations}

${ }^{1}$ Psychosocial Oncology Program, Peter MacCallum Cancer Centre, Melbourne, Victoria, Australia

${ }^{2}$ Melbourne School of Population and Global Health, University of Melbourne, Melbourne, Victoria, Australia

${ }^{3}$ Department of Clinical Haematology, Peter MacCallum Cancer Centre and Royal Melbourne Hospital, Melbourne, Victoria, Australia

${ }^{4}$ Australian Cancer Survivorship Centre, Peter MacCallum Cancer Centre, Melbourne, Victoria, Australia

${ }^{5}$ Sir Peter MacCallum Department of Oncology, University of Melbourne, Melbourne, Victoria, Australia

${ }^{6}$ School of Psychology, University of Queensland, Brisbane, Queensland, Australia

${ }^{7}$ The Australian Centre for Emotionally Focused Therapy, Brisbane, Queensland,

Australia

${ }^{8}$ Landscape of Life, Melbourne, Victoria, Australia

${ }^{9}$ Cancer Experiences Research, Peter MacCallum Cancer Centre, Melbourne, Victoria, Australia

Contributors BP, MF: contributed to design of proposed study and drafting and revising the paper. DR: contributed to design of proposed study and revising the paper. YP-H: contributed to design of proposed study, description of medical/ behavioural component of intervention and revising the paper. $\mathrm{MJ}$ : contributed to critical review of proposed study design and drafting of paper. TG: contributed to design of medical/behavioural component of intervention and revision of paper. CS: contributed to design of psychological component of intervention and revision of paper. JG: contributed to designing data collection tools, describing the psychological component of intervention and revising of paper. JP-N: contributed to design of proposed study and drafting and revising paper. $\mathrm{AD}$ : contributed to writing statistical analysis plan and revising of paper. SB: contributed to revising paper. SE: contributed to design of proposed study and revising paper.

Funding This work was supported by Foundation Grant from Peter MacCallum CancerFoundation, grant number (1839).

Competing interests None declared.

Patient and public involvement Patients and/or the public were involved in the design, or conduct, or reporting, or dissemination plans of this research. Refer to the Methods section for further details.

Patient consent for publication Not required.

Provenance and peer review Not commissioned; externally peer reviewed.

Open access This is an open access article distributed in accordance with the Creative Commons Attribution Non Commercial (CC BY-NC 4.0) license, which permits others to distribute, remix, adapt, build upon this work non-commercially, and license their derivative works on different terms, provided the original work is properly cited, appropriate credit is given, any changes made indicated, and the use is non-commercial. See: http://creativecommons.org/licenses/by-nc/4.0/.

ORCID iD

Brindha Pillay http://orcid.org/0000-0002-2142-1002

\section{REFERENCES}

1 Barbera L, Zwaal C, Elterman D, et al. Interventions to address sexual problems in people with cancer. Curr Oncol 2017;24:192-200.

2 Yi JC, Syrjala KL. Sexuality after hematopoietic stem cell transplantation. Cancer J 2009;15:57-64.

3 Dyer G, Gilroy N, Bradford J, et al. A survey of fertility and sexual health following allogeneic haematopoietic stem cell transplantation in New South Wales, Australia. Br J Haematol 2016;172:592-601.

4 Zantomio D, Grigg AP, MacGregor L, et al. Female genital tract graftversus-host disease: incidence, risk factors and recommendations for management. Bone Marrow Transplant 2006;38:567-72.

5 Thygesen $\mathrm{KH}$, Schjødt I, Jarden M. The impact of hematopoietic stem cell transplantation on sexuality: a systematic review of the literature. Bone Marrow Transplant 2012;47:716-24.
6 Candy B, Jones L, Vickerstaff V, et al. Interventions for sexual dysfunction following treatments for cancer in women. Cochrane Database Syst Rev 2016;2: :Cd005540.

7 El-Jawahri A, Fishman SR, Vanderklish J, et al. Pilot study of a multimodal intervention to enhance sexual function in survivors of hematopoietic stem cell transplantation. Cancer 2018;124:2438-46.

8 Besharat MA. Management strategies of sexual dysfunctions. J Contemp Psychother 2001;31:161-80.

9 Johnson SM. The practice of emotionally focused couple therapy: creating connection. Abingdon: Brunner-Routledge, 2004.

10 Beasley CC, Ager R. Emotionally focused couples therapy: a systematic review of its effectiveness over the past 19 years. J Evid Based Soc Work 2019:144-59.

11 Johnson SM, Simakhodskaya Z, Moran M. Addressing issues of sexuality in couples therapy: emotionally focused therapy meets sex therapy. Curr Sex Health Rep 2018;10:65-71.

12 Grayer J. Emotionally focused therapy for couples: a safe Haven from which to explore sex during and after cancer. Sexual and Relationship Therapy 2016;31:488-92.

13 Johnson S, Zuccarini D. Integrating sex and attachment in emotionally focused couple therapy. J Marital Fam Ther 2010;36:431-45.

14 Johnson SM, Hunsley J, Greenberg L, et al. Emotionally focused couples therapy: status and challenges. Clin Psychol 1999;6:67-79.

15 Novak JR, Sandberg JG, Stucki B, et al. A contextual analysis of high-fidelity and low-fidelity sessions in emotion-focused couples therapy. J Couple Relatsh Ther 2016;15:36-60.

16 Burgess Moser M, Johnson SM, Dalgleish TL, et al. Changes in Relationship-Specific attachment in emotionally focused couple therapy. J Marital Fam Ther 2016;42:231-45.

17 Wiebe SA, Elliott C, Johnson SM, et al. Attachment change in emotionally focused couple therapy and sexual satisfaction outcomes in a two-year follow-up study. J Couple Relatsh Ther 2019:18:1-21.

18 McLean LM, Walton T, Rodin G, et al. A couple-based intervention for patients and caregivers facing end-stage cancer: outcomes of a randomized controlled trial. Psychooncology 2013;22:28-38.

19 Johnson S. Hold me tight: seven conversations for a lifetime of love. New York: Little, Brown Spark, 2008.

20 Tulloch Het al. Healing hearts together: relationship education program: facilitator's guide for small groups. An adaptation of the hold me tight: conversations for connection relationship education program developed by Dr Sue Johnson. Ontario, Canada: ICEEFT \& University of Ottawa Heart Institute, 2017.

21 Kallos-Lilly V, Fitzgerald J. An emotionally focused workbook for couples: the two of us. New York and London: Routledge, 2015.

22 Kroenke K, Spitzer RL, Williams JB. The PHQ-9: validity of a brief depression severity measure. J Gen Intern Med 2001;16:606-13.

23 Spitzer RL, Kroenke K, Williams JBW, et al. A brief measure for assessing generalized anxiety disorder: the GAD-7. Arch Intern Med 2006;166:1092-7.

24 Brennan KA, Clark CL, Shaver PR. Self-report measure-ment of adult attachment: An integrative overview. In: Attachment theory and close relationships. New York: Guilford, 1998: 46-76.

25 Wei M, Russell DW, Mallinckrodt B, et al. The experiences in close relationship scale (ECR)-short form: reliability, validity, and factor structure. J Pers Assess 2007;88:187-204.

26 Fletcher GJO, Simpson JA, Thomas G. The measurement of perceived relationship quality components: a confirmatory factor analytic approach. Personality and Social Psychology Bulletin 2000;26:340-54.

27 Rosen RC, Riley A, Wagner G, et al. The International index of erectile function (IIEF): a multidimensional scale for assessment of erectile dysfunction. Urology 1997;49:822-30.

28 Rosen RC, Cappelleri JC, Gendrano N. The International index of erectile function (IIEF): a state-of-the-science review. Int J Impot Res 2002;14:226-44.

29 Rosen R, Brown C, Heiman J, et al. The female sexual function index (FSFI): a multidimensional self-report instrument for the assessment of female sexual function. J Sex Marital Ther 2000;26:191-208.

30 Sandberg JG, Brown AP, Schade LC, et al. Measuring fidelity in emotionally focused couples therapy (EFT): a pilot test of the EFT therapist fidelity scale. Am J Fam Ther 2015;43:251-68.

31 Denton WH, Johnson SM, Burleson BR. Emotion focused therapytherapist fidelity scale (EFT-TFS): conceptual development and content validity. J Couple Relatsh Ther 2009;8:226-46. 